\title{
A Critical Analysis of the Assumptions of Type D Personality: Comparing Prediction of Health-Related Variables with the Five Factor Model
}

\author{
Sharon Horwood; Jeromy Anglim ${ }^{1}$
}

\begin{abstract}
The present study critically evaluated several assumptions related to Type D personality within the context of predicting health-related variables. Specifically, it compared categorical with continuous representations of Type D personality, assessed evidence for interactive effects of the Type D subscales (negative affectivity and social inhibition), and compared the predictive validity of Type D personality with the Big Five. Healthy adults $(\mathrm{n}=224)$ completed the DS14 and the NEO-PI-R, as well as measures of health behaviors, social support, physical symptoms, and psychological symptoms. Categorical Type D had much poorer prediction than the continuous subscales of social inhibition and negative affectivity, and there was no interaction between the subscales. While negative affectivity and social inhibition were effective at predicting health-related variables, the prediction was equivalent to that achieved by neuroticism and extraversion. Conscientiousness was found to be an important predictor that was missing from Type $\mathrm{D}$. There are reasons to prefer the use of the Big Five over Type D personality when the aim is to predict health-related variables. Where the DS14 is used, it is recommended to also include a measure of conscientiousness.

Keywords: personality, Type D personality, health determinants, five-factor model, narrow traits
\end{abstract}

\section{Introduction}

Type D personality is defined as the presence of high levels of both negative affectivity and social inhibition, and represents a personality typology that denotes a propensity to experience elevated levels of psychological distress (Denollet, 2005). Theory and research suggests that Type D personality is a risk factor for

\section{${ }^{1}$ Citation Information:}

Horwood, S., \& Anglim, J. (2017). A Critical Analysis of the Assumptions of Type D Personality: Comparing Prediction of Health-Related Variables with the Five Factor Model. Personality and Individual Differences, 117, 172-176.

http://dx.doi.org/10.1016/j.paid.2017.06.001

Please see the doi to publisher's copy of record.

Sharon Horwood and Jeromy Anglim, Deakin University, Geelong, Australia, School of Psychology. Data, scripts, and materials for this study are available at https://osf.io/vb4ve. Correspondence concerning this article should be addressed to Sharon Horwood, School of Psychology, Deakin University, Locked Bag 20000, Geelong, 3220, Australia. Email: sharon.horwood@ deakin.edu.au 
increased morbidity and mortality in several chronic illnesses including coronary artery disease (Svansdottir et al., 2013) and type 2 diabetes (Nefs et al., 2015). Various mechanisms have been proposed to explain the relationship between Type D and chronic illness, including greater levels of negative health behaviors (Horwood, Anglim, \& Tooley, 2016), lower levels of social functioning (Grande, Romppel, Michal, \& Brähler, 2014), and a tendency to adopt poorer coping strategies (Booth \& Williams, 2015).

Nonetheless, it is unclear whether using the domain-specific Type D measure of personality offers any benefits over a general personality framework like the Big Five (i.e., extraversion, neuroticism, conscientiousness, agreeableness, openness) (Digman, 1990). Furthermore, there are various active methodological debates that are relevant to the inclusion of personality into models of health behaviors. These include (a) whether personality should be conceptualized categorically in terms of personality types (Ferguson et al., 2009), (b) whether traits have interactive effects on criteria (Atherton, Robins, Rentfrow, \& Lamb, 2014), and (c) the extent to which narrow personality facets incrementally improve prediction over and above broad personality factors (Anglim \& Grant, 2014). The Type D personality construct implies that personality types are meaningful and useful, traits have interactive effects, and that the narrow traits of negative affectivity and social inhibition are more relevant than broad traits such as neuroticism and extraversion. While a few studies have challenged the assumptions of Type D personality regarding its status as a categorical variable (Ferguson et al., 2009; Howard \& Hughes, 2012; Kelly-Hughes, Wetherell, \& Smith, 2014) and its interactive effects (Horwood et al., 2016; Kelly-Hughes et al., 2014), almost no research has examined the ability of Type D personality to predict health-related variables in comparison to a comprehensive hierarchical framework of personality based on the Big Five. Thus, the present study sought to address these issues by comparing the predictive validity of various representations of Type D personality with the Five-Factor Model (Costa \& McCrae, 1992). Specifically, it focused on the ability to predict the four health-related outcome variables of health behaviors, social support, physical symptoms, and psychological symptoms.

\section{Challenges to the Assumptions Underpinning Type D Personality}

Several assumptions of Type D personality have been challenged in the personality literature. First, Type D personality is a dichotomous variable scored using cut-off scores for the subscales of negative affectivity and social inhibition. Treating traits as dichotomous variables is contrary to current thinking in personality literature and taxonomic research (Ferguson et al., 
2009; Kelly-Hughes et al., 2014). Research shows that dichotmizing an interval scale reduces correlations (Aguinis, Pierce, \& Culpepper, 2009; Rucker, McShane, \& Preacher, 2015). Demonstrating this general point, Horwood et al. (2016) found that treating Type $\mathrm{D}$ as a dichotomous variable reduced predictive validity relative to a continuous representation of Type D.

Second, the scoring of Type $\mathrm{D}$ personality requires an individual to score above a threshold on both negative affectivity and social inhibition. Consistent with how Type D is theorized to operate, this multiple hurdle approach implies that the two subscales have an interactive effect over and above the main effects (Denollet, Pedersen, Vrints, \& Conraads, 2013). Given the overlap of the Big Five traits of neuroticism (+) and extraversion (-) with negative affectivity and social inhibition respectively, if Type D theory was correct, then we might also expect interaction effects to be present between neuroticism and extraversion. There is almost no evidence that traits such as neuroticism and extraversion have interactive effects (Atherton et al., 2014), nor is there evidence that the two subscales of negative affectivity or social inhibition have interactive effects in predicting health-related variables (Horwood et al., 2016; Kelly-Hughes et al., 2014).

Third, the traits that underpin Type D Personality (negative affectivity and social inhibition) are assumed to be sufficiently different to other well-known constructs (i.e. neuroticism and extraversion) that the measure can be considered unique. This assumption has been criticized in the literature (Coyne \& de Voogd, 2012). A meta-analysis (Horwood, Anglim, \& Tooley, 2015) found relatively large mean correlations between negative affectivity and neuroticism of $r=.74$ and between social inhibition and extraversion of $r=-.63$.

\section{Type D Personality and the Big Five as Predictors of Health Outcomes}

There is substantial evidence that Big Five personality traits such as neuroticism and extraversion can predict behavioral risk factors for chronic illness such as excess weight (Armon, Melamed, Shirom, Shapira, \& Berliner, 2013), tobacco smoking (Cheng \& Furnham, 2016), and alcohol and drug abuse (Lackner, Unterrainer, \& Neubauer, 2013). Likewise, conscientiousness is a strong predictor of healthy behaviors such as commitment to a healthy lifestyle (Lodi-Smith et al., 2010) abstaining from alcohol consumption (Roberts, Walton, \& Bogg, 2005), and adhering to long-term health goals (Booth-Kewley \& Vickers, 1994). Not surprisingly given the overlap with neuroticism and extraversion, Type D research has shown relationships with health behaviors (e.g. adequate physical activity, Nefs et al., 2015) and social support (Williams \& Wingate, 2012). Nonetheless, the lack of 
overlap with conscientiousness in particular is likely to yield poorer prediction of health behaviors.

\section{The Current Study}

Collectively, these issues raise a number of question about the utility of Type D personality in predictive models of healthrelated variables. The present study sought to address these issues by comparing the validity of various representations of Type D personality with the Five-Factor Model. Both Type D personality and the Big Five were used to predict health behaviors, social support, physical symptoms and psychological symptoms. Health behaviors and social support are two theorized mechanisms by which Type D personality is expected to influence health outcomes, and physical and psychological symptoms provide a measure of health outcomes. The study examined four assumptions related to Type D personality: (a) that personality traits have an interactive effect that is greater than the main effects, (b) that the effect of traits are discontinuous (as implied by cut-off scores on negative affectivity and social inhibition), (c) that negative affectivity and social inhibition are the most important personality traits for predicting health-related variables, and (d) using the specific traits of negative affectivity and social inhibition is better or at least as good as using the big five.

\section{Method}

Item-level data, the data analysis script, and selected materials are available from the Open Science Framework at https://osf.io/vb4ve.

\section{Participants and Procedure}

Participants were recruited through online forums including university websites, social media, and online health and wellbeing forums. Participants completed measures of demographics, personality, and the health-related variables: i.e., health behaviors, social support, physical symptoms, and psychological symptoms. After removing nine cases due to missing data or careless responding, the final sample consisted of 244 adults (76\% female) with ages ranging from 18 to 70 years $(M=32.6, S D=12.8)$. Almost all participants resided in Australia. A previous study that used this sample examined the correlations between Type D and the factors and facets of the NEO-PI-R, but this previous study did not examine health-related variables and did not compare the predictive validity of different models of personality (Horwood et al., 2015). 


\section{Measures}

Type D Personality.

The DS14 is the original and standard measure of Type D personality (Denollet, 2005). The measure consists of two 7-item subscales, negative affectivity and social inhibition, which are scored as the sum of respective items after any necessary item reversal. Each item is rated on a 5 -point scale from $0=$ false to $4=$ true. An individual is classified as having Type D personality if they score 10 or more on both subscales (Denollet, 2005). A continuous Type D scale was also calculated as the sum of social inhibition and negative affectivity.

Personality.

The NEO Personality Inventory - Revised (NEO-PI-R) is a 240-item measure of the Big Five factors each with six nested facets (Costa \& McCrae, 1992). Items are rated on a 5-point scale ranging from $1=$ strongly disagree to $5=$ strongly agree. Factor and facet scores were obtained by taking the mean of constituent items after any necessary item reversal.

Health Behaviors.

The General Preventive Health Behaviors Checklist (Amir, 1987) measures preventative health behaviors and provides a global index of health behavior. Each item is originally rated on a 3-point scale: $0=$ do not do it, $1=$ sometimes do it, and $2=$ yes always, or almost always do it. A health behavior score was computed as the mean of these seven items.

Social Support.

The Quality of Social Network and Social Support Scale (Dalgard, Bjørk, \& Tambs, 1995) was used to measure perceived social support. Following Williams et al (2008), the support from neighbors subscale was excluded, leaving nine items that measured perceived support experienced from friends and family. An overall social support score was formed by taking the mean of all items.

Physical and Psychological Symptoms.

The Rotterdam Symptom Checklist (De Haes, 1990) is a 35 item scale that measures the number of self-reported physical and psychological symptoms a person has experienced in the previous week. The measure uses a 4 -item response scale where $1=$ not at all, $2=$ a little, $3=$ moderately, and $4=$ very much. Scores for symptom severity are represented as the sum of items.

\section{Data Analysis}

To examine the bivariate relationship between personality and health-related variables, correlations of Type D and Big Five personality with health-related variables were obtained. In several instances mean correlations were computed averaging across the 
four health-related variables. To ensure that positive correlations reflected healthier outcomes, the correlations with physical and psychological symptoms was reversed when computing this average. To provide an overall assessment of the predictive validity of different representations of Type D and the Five-Factor Model, a series of regression models were estimated that used different personality predictors. Several regression models examined interactions between personality traits. Interaction terms were included by taking the product of the constituent predictor variables. Adjusted multiple correlation (i.e., the square root of adjusted r-squared) was reported for each model for each set of variables as an index of prediction. It provides an unbiased assessment of relative prediction in the population, which is important given the varying numbers of predictors (Anglim \& Grant, 2014, 2016). In addition, estimates and confidence intervals of incremental population variance explained were also obtained using the double-adjusted R-squared bootstrap method (Anglim \& Grant, 2014). For completeness, we present facet-level correlations with health outcomes in the online supplement.

\section{Results}

\section{Personality and Health-related Variables}

Table 1 shows the correlations of Big Five and Type D personality with the health-related variables. As expected, there was a strong negative correlation between social inhibition and extraversion ( $r=-.77$, corrected for reliability $r=.-.87)$ and strong positive correlation between neuroticism and negative affectivity ( $r$ $=76$, corrected for reliability $r=.84$ ). Likewise, the mean correlation with health-related variables (after reversing physical and psychological symptoms) for negative affectivity (mean $r=-$ .50) were very similar to those for neuroticism (mean $r=-.52$ ), and those for social inhibition (mean $r=-.38$ ) were similar but the reverse of those for extraversion (mean $r=.34$ ). Correlations for the Continuous Type D scale (mean $r=-.51$ ) were similar to negative affectivity and neuroticism. After neuroticism, the largest correlations with health-related variables were observed for conscientiousness (mean $r=.36$ ) and extraversion (mean $r=.34$ ), followed by agreeableness (mean $r=.16$ ) and openness (mean $r=$ $.04)$.

In terms of the Big Five predicting health behavior, the strongest correlate was conscientiousness $(r=.51)$, followed by neuroticism $(r=-.36)$ and extraversion $(r=.28)$. Social support reflected a mix of both neuroticism $(r=-.50)$ and extraversion $(r=$ .47) along with moderate correlations with both agreeableness ( $r=$ $.35)$ and conscientiousness $(r=.35)$. Physical symptoms had strong correlations with neuroticism $(\mathrm{r}=.49)$, and consistent with 
construct overlap, psychological symptoms was highly correlated with neuroticism $(r=.71)$.

Table 1

Descriptive Statistics, Reliability, and Intercorrelations between Correlations between the Big Five, Type D Personality, and Health-Related Variables

\begin{tabular}{|c|c|c|c|c|c|c|c|c|c|c|c|c|c|c|c|}
\hline Variable & $\mathrm{M}$ & SD & $\alpha$ & 1 & 2 & 3 & 4 & 5 & 6 & 7 & 8 & 9 & 10 & 11 & 12 \\
\hline \multicolumn{16}{|l|}{ Outcomes } \\
\hline 1. Health Behaviors & 1.16 & 0.42 & .65 & & & & & & & & & & & & \\
\hline 2. Social Support & 12.40 & 3.78 & .79 & 30 & & & & & & & & & & & \\
\hline 3. Physical Symptoms & 1.51 & 0.39 & .89 & -.26 & -.35 & & & & & & & & & & \\
\hline 4. Psychological Symptoms & 2.07 & 0.74 & .93 &.,- 32 &.,- 46 & .69 & & & & & & & & & \\
\hline \multicolumn{16}{|l|}{ Big 5} \\
\hline 5. Agreeableness & 3.55 & 0.41 & .90 & .08 & $\mathbf{3 5}$ & $=, 03$ & -.20 & & & & & & & & \\
\hline 6. Conscientiousness & 3.36 & 0.44 & .91 & 51 & 35 & -.29 & -.30 & .09 & & & & & & & \\
\hline 7. Extraversion & 3.25 & 0.47 & .91 & .28 & .47 & -.28 & -.32 & .12 & .26 & & & & & & \\
\hline 8. Neuroticism & 3.02 & 0.54 & .93 & -.36 & -.50 & .49 & .71 &.- .26 & -.42 & -.44 & & & & & \\
\hline 9. Openness & 3.50 & 0.41 & .89 & .11 &, 05 &,- 03 &, 03 & .24 & .10 & .30 & -.06 & & & & \\
\hline \multicolumn{16}{|l|}{ Type D } \\
\hline 10. Negative Affectivity & 11.62 & 6.27 & .89 &.,- 31 & -.50 & .48 & .73 &.,- 34 & -.32 & -.45 & .76 &,- 06 & & & \\
\hline 11. Social Inhibition & 11.34 & 6.12 & .86 & -.27 & -.49 & .35 & .42 & -.24 & -.23 & -.77 & .56 & -.24 & 54 & & \\
\hline 12. Type D Continuous & 22.96 & 10.86 & & -.33 & -.56 & .47 & .66 & -.33 & -.31 & -.69 & .76 & -.17 & .88 & .87 & \\
\hline 13. Type D Categorical & 0.46 & 0.50 & & -.15 & -.43 & .39 & .52 & -.29 & -.19 & -.52 & .63 & -.11 & .74 & .65 & .80 \\
\hline
\end{tabular}

Note. Reliability is Cronbach's alpha. $|r| \geq .20$ are in bold. $|r| \geq .13$ are statistically significant ( $p$ $<.05)$.

\section{Predictive Models}

In order to compare the degree to which different models of personality can predict health behaviors, a set of regressions were performed. Table 2 shows the adjusted multiple correlations from models predicting each health-related variable from different sets of predictors. We initially examined different representations of Type D personality: (a) a categorical version of Type D, (b) negative affectivity and social inhibition as main effects, and (c) negative affectivity and social inhibition as main effects along with the interaction of negative affectivity and social inhibition. Table 3 presents estimates of population incremental variance explained as well as $95 \%$ double-adjusted-R-squared confidence intervals (Anglim \& Grant, 2014).

Categorical Type D had substantially weaker prediction than treating the two subscales separately as main effects (average adjusted multiple $R$ of .37 versus .52). In an analysis presented in the online supplement, we examined whether using different cutoff scores on NA and SI for generating categorical Type D resulted in improved predictive validity. While this exploratory analysis was able to achieve mean adjusted multiple $R$ of .45 using cut-offs of 9 for SI and 15 for NA, most of this improvement in predictive validity is likely to be both sample-specific and due to capitalizing 
Table 2

on multiple testing. We also ran supplemental analysis (see OSF repository) examining regression models that included scale scores for SI and NA as well as categorical Type D. Categorical Type D only improved prediction for health behaviors (increase in adjusted multiple $R=.049, p=.001$ ).

Adjusted Multiple Correlation Coefficients for Linear Regressions predicting Health-Related Variables from Different Sets of Type D and NEO-PI-R Personality Predictors

\begin{tabular}{lcccccc}
\hline Predictors & $k$ & HB & SS & PHYS & PSYCH Mean $R$ \\
\hline Type D & & & & & & \\
$\quad$ Type D Categorical & 1 & .13 & .43 & .39 & .51 & .37 \\
Type D Continuous & 1 & .32 & .56 & .47 & .66 & .50 \\
NA + SI & 2 & .32 & .56 & .48 & .73 & .52 \\
NA + SI + NA*SI & 3 & .32 & .56 & .48 & .72 & .52 \\
NEO & & & & & & \\
E + N & 2 & .37 & .56 & .49 & .71 & .54 \\
E + N + E*N & 3 & .38 & .56 & .50 & .71 & .54 \\
Big Five Factors & 5 & .54 & .63 & .50 & .71 & .59 \\
Big Five Factors + two-way interactions & 15 & .54 & .63 & .50 & .72 & .60 \\
30 Facets & 30 & .60 & .66 & .53 & .78 & .64 \\
\hline
\end{tabular}

Note. Cell values represent the square root of adjusted $R$-squared. $k$ is the number of predictors in the regression model. Mean $R$ represents mean adjusted multiple $\mathrm{r}$ for the predictor set averaged over health-related variables. NA $=$ negative affectivity, $\mathrm{SI}=$ social inhibition, $\mathrm{E}=$ extraversion, $\mathrm{N}=$ neuroticism, $\mathrm{HB}=$ health behaviors, $\mathrm{SS}=$ social support, $\mathrm{PHYS}=$ physical symptom severity, PSYCH = psychological symptom severity. Representations with both + and * terms represent main effects and interaction terms respectively.

The assumption that Type D involves an interactive effect of negative affectivity and social inhibition was examined. Results indicated that the interaction of negative affectivity and social inhibition did not improve prediction over and above main effects of negative affectivity and social inhibition.

Predictive models using negative affectivity and social inhibition as predictors were compared to models using neuroticism and extraversion as predictors. Overall, prediction was very similar, although prediction of health behaviors was slightly larger when using extraversion and neuroticism. Similar to the results with Type $\mathrm{D}$, including the interaction of neuroticism and extraversion did not improve prediction. A further test, where all ten two-way interactions between the Big Five were added, indicated that including interactions did not improve prediction.

We then examined whether including all Big Five factors improved prediction of health-related variables over just 
neuroticism and extraversion. Consistent with the strong correlations for conscientiousness, and to some extent, agreeableness, prediction was larger for the Big Five factors compared to the model that only included neuroticism and extraversion in predicting health behavior (mean adjusted multiple $R=.54$ versus .37) and social support (mean adjusted multiple $R=$ .63 versus .56), but not for physical or psychological symptoms. Finally, models that included the 30 facets as predictors of healthrelated variables indicated a modest but meaningful increase in prediction over and above the Big Five (i.e., mean adjusted multiple $R$ of .64 versus .59), although only psychological symptoms had confidence intervals that excluded zero.

Table 3

Estimate of Population Incremental Variance Explained and Confidence intervals (95\%) for Incremental Variance Explained of Regression Models with Predictor Set 2 over Predictor Set 1

\begin{tabular}{|c|c|c|c|c|c|}
\hline Set 1 & Set 2 & $\begin{array}{c}\mathrm{HB} \\
\Delta \rho^{2}[95 \% \mathrm{Cl}]\end{array}$ & $\begin{array}{c}\text { SS } \\
\Delta \rho^{2}[95 \% \mathrm{Cl}]\end{array}$ & $\begin{array}{c}\text { PHYS } \\
\Delta \rho^{2}[95 \% \mathrm{Cl}]\end{array}$ & $\begin{array}{c}\text { PSYCH } \\
\Delta \rho^{2}[95 \% \mathrm{Cl}]\end{array}$ \\
\hline Type D Cat & $\mathrm{NA}+\mathrm{SI}$ & $.084[.033, .139]$ & $.129[.053, .198]$ & $.083[.027, .147]$ & $.264[.186, .339]$ \\
\hline $\mathrm{NA}+\mathrm{SI}$ & $\mathrm{NA}+\mathrm{SI}+\mathrm{NA}^{*} \mathrm{SI}$ & $.001[-.008, .040]$ & $.005[-.006, .027]$ & $-.002[-.007, .013]$ & $-.002[-.004, .005]$ \\
\hline $\mathrm{NA}+\mathrm{SI}$ & $\mathrm{E}+\mathrm{N}$ & $.039[-.006, .088]$ & $.007[-.057, .077]$ & $.008[-.066, .086]$ & $-.026[-.115, .062]$ \\
\hline $\mathrm{NA}+\mathrm{SI}$ & Big Five & $.185[.101, .276]$ & $.088[.018, .162]$ & $.017[-.069, .105]$ & $-.025[-.117, .065]$ \\
\hline $\mathrm{E}+\mathrm{N}$ & Big Five & $.146[.075, .221]$ & $.082[.029, .145]$ & $.009[-.016, .059]$ & $.001[-.011, .027]$ \\
\hline Big Five & Big Five + Interactions & $.001[-.045, .070]$ & $-.002[-.034, .043]$ & $.001[-.041, .054]$ & $.018[-.017, .065]$ \\
\hline Big Five & 30 Facets & $.074[-.001, .167]$ & $.039[-.016, .106]$ & $.033[-.045, .153]$ & $.104[.044, .169]$ \\
\hline
\end{tabular}

Note. The estimate of $\Delta \rho^{2}$ is the increase in adjusted $\mathrm{r}$-squared from the regression with predictor set 1 to predictor set 2 . Confidence intervals that do not include zero are bolded. Predictors correspond to those mentioned in Table 2. Interactions refers to all two-way interactions between Big Five. $\mathrm{NA}=$ negative affectivity, $\mathrm{SI}=$ social inhibition, $\mathrm{E}=$ extraversion, $\mathrm{N}=$ neuroticism, $\mathrm{HB}=$ health behaviors, $\mathrm{SS}=$ social support, $\mathrm{PHYS}=$ physical symptom severity, $\mathrm{PSYCH}=$ psychological symptom severity.

\section{Discussion}

The current study aimed to critically evaluate assumptions of Type D personality and compare prediction of health-related variables with the Big Five. Findings have implications for the understanding of Type D personality and the value of using a domain-specific versus a general model of personality to predict health-related variables.

\section{Assumptions of Type D}

Treating Type D as a categorical variable substantially reduced predictive validity compared to using the interval subscales or using a continuous sum score. Thus, Type D personality is not immune to the usual consequences of dichotomizing interval scales 
(Aguinis et al., 2009). Categorical Type D was also only able to improve prediction, albeit modestly, for one of the four outcomes, over and above continuous subscales. Consistent with past research (Atherton et al., 2014; Horwood et al., 2016; Kelly-Hughes et al., 2014), results also showed no evidence of interactive effects of negative affectivity and social inhibition. Thus, while interactive effects are one means of personality types providing superior prediction to interval scales (Asendorpf, 2003), this does not appear to be the case when using either the interaction of interval subscales or when using categorical Type D.

Further analyses examining optimal cut-offs suggested that for the outcomes studied, negative affectivity was more relevant than social inhibition. Thus, similar prediction was achieved by relying entirely on a cut-off on negative affectivity. This is consistent with negative affectivity (or neuroticism) having a substantial influence on emotional experience and symptoms appraisal while social inhibition (or extraversion) has a greater influence on social processes.

\section{Type D Personality and the Big Five}

Compared to Type D personality, the Big Five provided a superior framework for predicting health-related variables. Absolute correlations between neuroticism and negative affectivity and between extraversion and social inhibition were above .70, and above .80 when adjusting for reliability. Thus, unsurprisingly, negative affectivity and social inhibition achieved similar predictive validity to neuroticism and extraversion. However, the lack of conscientiousness in the Type D framework was a particular shortcoming. The importance of conscientiousness in predicting health behavior is well established in the literature (HaggerJohnson \& Whiteman, 2007; Roberts et al., 2005). Thus, while neuroticism and negative affectivity both yielded similarly good prediction of variables related to negative psychological symptoms, conscientiousness was particularly important in predicting health behaviors that imply discipline and personal control, such as not smoking, reduced alcohol consumption, and obtaining regular medical check-ups. More generally, using the Big Five terminology of extraversion and neuroticism allows for theoretical links to be made with an extensive Big Five literature.

\section{Implications for Health Professionals}

While personality types yield inferior prediction, it is worth considering whether a type-approach provides benefits to health professionals (Asendorpf, 2003; Asendorpf \& Denissen, 2006). Health professionals often need to make and justify categorical decisions. Classifying a patient as Type D may be used to justify different treatment protocols and may lead to referrals for other services to assist with treatment adherence. Categorization also 
aligns with the traditional model of disease classification, and may be simpler for health professionals and patients to understand.

Nonetheless, even when taking a sympathetic perspective to personality types, the present research has several implications for health practitioners. First, where applications involve developing predictive models of morbidity or mortality, interval scales will almost always yield better prediction. Second, a more robust assessment of personality could be achieved by using a complete Big Five instrument. In particular, the addition of conscientiousness would be particularly beneficial for identifying general dispositions relevant to health and treatment adherence behaviors. As testing duration would often be an issue, a test like the 20-item mini-IPIP might provide an appropriate trade-off between reliability and convenience (Donnellan, Oswald, Baird, \& Lucas, 2006). Future research could examine ways of making information from a measure of the Big Five more relevant to clinical decision-making. Such an approach could combine interval scale scores with various categorical classifications; however, a more nuanced typology maybe useful, particularly, where conscientiousness forms part of any such framework.

\section{Limitations and Future Research}

This study had several limitations that should be noted. First, the study relied on self-reported health-related variables. Using more objective measures of physical and psychological symptoms would likely result in reduced correlations. Second, the study used a general population rather a population experiencing a specific illness. Medical applications of Type D personality are often concerned with particular illness groups. Third, the current study only used a subset of possible health-outcome measures relevant to Type D personality. While health behaviors and social support are important considerations, treatment adherence, morbidity and mortality are particularly relevant in health contexts.

\section{Conclusion}

The present study aimed to critically evaluate a number of assumptions regarding the predictive validity of Type D personality. Many of these assumptions are not consistent with contemporary personality theory and were not supported by the data. Type D personality captures two important narrow traits relevant to predicting health-related variables, however the Big Five provided a more nuanced framework for understanding connections between personality and health-related variables. Thus, in general, we recommend that where possible researchers use the Big Five instead of Type D personality. If Type D personality is to be used, then we strongly encourage that researchers and practitioners also include a measure of conscientiousness. 


\section{References}

Aguinis, H., Pierce, C. A., \& Culpepper, S. A. (2009). Scale coarseness as a methodological artifact: Correcting correlation coefficients attenuated from using coarse scales. Organizational Research Methods, 12(4), 623652.

Amir, D. (1987). Preventive behaviour and health status among the elderly. Psychology and Health, 1(4), 353-378.

Anglim, J., \& Grant, S. (2014). Incremental criterion prediction of personality facets over factors: Obtaining unbiased estimates and confidence intervals. Journal of Research in Personality, 53(0), 148-157. doi:http://dx.doi.org/10.1016/j.jrp.2014.10.005

Anglim, J., \& Grant, S. (2016). Predicting Psychological and Subjective Well-Being from Personality: Incremental Prediction from 30 Facets Over the Big 5. Journal of Happiness Studies, Online First. doi:10.1007/s10902014-9583-7

Armon, G., Melamed, S., Shirom, A., Shapira, I., \& Berliner, S. (2013). Personality traits and body weight measures: Concurrent and across - time associations. European Journal of Personality, 27(4), 398-408. doi:10.1002/per.1902

Asendorpf, J. (2003). Head - to - head comparison of the predictive validity of personality types and dimensions. European Journal of Personality, 17(5), 327-346.

Asendorpf, J., \& Denissen, J. (2006). Predictive Validity of Personality Types Versus Personality Dimensions From Early Childhood to Adulthood: Implications for the Distinction Between Core and Surface Traits. MerrillPalmer Quarterly, 52(3), 486-513. doi:10.1353/mpq.2006.0022

Atherton, O. E., Robins, R. W., Rentfrow, P. J., \& Lamb, M. E. (2014). Personality correlates of risky health outcomes: findings from a large Internet study. Journal of Research in Personality, 50, 56-60.

Booth, L., \& Williams, L. (2015). Type D personality and dietary intake: The mediating effects of coping style. Journal of Health Psychology, 20(6), 921-927. doi:10.1177/1359105315573433

Booth-Kewley, S., \& Vickers, R. R. (1994). Associations between major domains of personality and health behavior. Journal of Personality, 62(3), 281-298. doi:10.1111/j.1467-6494.1994.tb00298.x

Cheng, H., \& Furnham, A. (2016). The Big-Five personality traits, maternal smoking during pregnancy, and educational qualifications as predictors of tobacco use in a nationally representative sample. PloS One, $11(1)$.

Costa, P., \& McCrae, R. (1992). Revised NEO Personality Inventory.

Coyne, J., \& de Voogd, J. (2012). Are we witnessing the decline effect in the Type D personality literature? What can be learned? Journal of Psychosomatic Research, 73(6), 401-407.

Dalgard, O. S., Bjørk, S., \& Tambs, K. (1995). Social support, negative life events and mental health. The British Journal of Psychiatry, 166, 29-34. doi:10.1192/bjp.166.1.29

De Haes, J., \& Van Knippenberg, F. (1990). Rotterdam Symptom Checklist. Oncology, 4(5), 44-45.

Denollet, J. (2005). DS14: standard assessment of negative affectivity, social inhibition, and Type D personality. Psychosomatic Medicine, 67(1), 89-97. doi:10.1097/01.psy.0000149256.81953.49

Denollet, J., Pedersen, S. S., Vrints, C. J., \& Conraads, V. M. (2013). Predictive value of social inhibition and negative affectivity for cardiovascular events and mortality in patients with coronary artery disease: the Type D personality construct. Psychosomatic Medicine, 75(9), 873-881. doi:10.1097/PSY.0000000000000001

Digman, J. M. (1990). Personality structure: Emergence of the five-factor model. Annual Review of Psychology, 4l(1), 417-440.

Donnellan, M. B., Oswald, F. L., Baird, B. M., \& Lucas, R. E. (2006). The Mini-IPIP Scales: Tiny-yet-effective measures of the Big Five Factors of Personality. Psychological Assessment, 18(2), 192-203. doi:10.1037/1040-3590.18.2.192

Ferguson, E., Williams, L., O'Connor, R. C., Howard, S., Hughes, B. M., Johnston, D. W., . . Grealy, M. A. (2009). A taxometric analysis of type-D personality. Psychosomatic Medicine, 71(9), 981-986.

Grande, G., Romppel, M., Michal, M., \& Brähler, E. (2014). The Type D construct: Is social inhibition more than social fear? European Journal of Psychological Assessment, 30(4), 283-288. doi:10.1027/1015$5759 / \mathrm{a} 000189$

Hagger-Johnson, G. E., \& Whiteman, M. C. (2007). Conscientiousness facets and health behaviors: A latent variable modeling approach. Personality and Individual Differences, 43(5), 1235-1245. doi:10.1016/j.paid.2007.03.014 
Horwood, S., Anglim, J., \& Tooley, G. (2015). Type D personality and the Five-Factor Model: A facet-level analysis. Personality and Individual Differences, 83, 50-54.

Horwood, S., Anglim, J., \& Tooley, G. (2016). Statistically modelling the relationships between Type D personality and social support, health behaviors and symptom severity in chronic illness groups. Psychology \& Health, 31, 1047-1063.

Howard, S., \& Hughes, B. M. (2012). Construct, concurrent and discriminant validity of Type D personality in the general population: Associations with anxiety, depression, stress and cardiac output. Psychology \& Health, 27(2), 242-258.

Kelly-Hughes, D. H., Wetherell, M. A., \& Smith, M. A. (2014). Type D personality and cardiovascular reactivity to an ecologically valid multitasking stressor. Psychology and Health, 29(10), 1156-1175.

Lackner, N., Unterrainer, H.-F., \& Neubauer, A. C. (2013). Differences in Big Five personality traits between alcohol and polydrug abusers: Implications for treatment in the therapeutic community. International Journal of Mental Health and Addiction, 11(6), 682-692. doi:10.1007/s11469-013-9445-2

Lodi-Smith, J., Jackson, J., Bogg, T., Walton, K., Wood, D., Harms, P., \& Roberts, B. W. (2010). Mechanisms of health: education and health-related behaviours partially mediate the relationship between conscientiousness and self-reported physical health. Psychology \& Health, 25(3), 305-319. doi:10.1080/08870440902736964

Nefs, G., Speight, J., Pouwer, F., Pop, V., Bot, M., \& Denollet, J. (2015). Type D personality, suboptimal health behaviors and emotional distress in adults with diabetes: Results from Diabetes MILES-The Netherlands. Diabetes Research and Clinical Practice, 108(1), 94-105. doi:10.1016/j.diabres.2015.01.015

Roberts, B. W., Walton, K. E., \& Bogg, T. (2005). Conscientiousness and health across the life course. Review of General Psychology, 9(2), 156.

Rucker, D. D., McShane, B. B., \& Preacher, K. J. (2015). A researcher's guide to regression, discretization, and median splits of continuous variables. Journal of Consumer Psychology, 25(4), 666-678.

Svansdottir, E., Denollet, J., Thorsson, B., Gudnason, T., Halldorsdottir, S., Gudnason, V., . . . Karlsson, H. D. (2013). Association of type D personality with unhealthy lifestyle, and estimated risk of coronary events in the general Icelandic population. European Journal Of Preventive Cardiology, 20(2), 322-330. doi: $10.1177 / 2047487312441723$

Williams, L., O'Connor, R., Howard, S., Hughes, B., Johnston, D., Hay, J., . . . O'Carroll, R. (2008). Type-D personality mechanisms of effect: The role of health-related behavior and social support. Journal of Psychosomatic Research, 64(1), 63-69.

Williams, L., \& Wingate, A. (2012). Type D personality, physical symptoms and subjective stress: The mediating effects of coping and social support. Psychology \& Health, 27(9), 1075-1085. doi:10.1080/08870446.2012.667098 


\section{Online Supplement}

In addition to the Big Five, most personality researchers conceptualize personality traits hierarchically (Anglim \& Grant, 2014). The Five-Factor model, composed of five broad domains each containing six narrow facets, has arguably been the most influential (Costa \& McCrae, 1992). A meta-analysis (2004) found that a number of conscientiousness facets were related to specific health behaviors and outcomes. For example, the conscientiousness facets of self-control and responsibility were found to be strong predictors of alcohol use, drug use, and unhealthy eating. These and other facet-level studies have demonstrated the often meaningful incremental prediction that narrow traits can make (Anglim \& Grant, 2014; Paunonen \& Ashton, 2001). Given that the Type D construct consists of two relatively narrow traits (negative affectivity and social inhibition), comparing the predictive utility of Type D personality with the 30 facets that underpin the Big Five factors may be particularly useful.

To further explore which facets were important in predicting health-related variables, correlations between 30 personality facets and health-related variables were obtained. Table $\mathrm{S} 1$ presents descriptive statistics, and Table S2 presents the zeroorder correlations and semi-partial correlations where personality facets are adjusted for overlap with the Big Five factors. Zero-order correlations indicate strength of relationships in terms of the intrinsic meaning of the scale, whereas semi-partial correlations examine what is unique about the facet-outcome relationship over and above the Big Five. In general, larger facet correlations tended to cluster together within factors. The largest zero-order correlations for facets were observed for depression and anxiety which seemed to largely driven by construct overlap with psychological symptoms. They also provided a fairly pure measure of negative affective symptoms that were also relevant to other scales. However, strong facet-level predictors of health behaviors included several conscientiousness facets including self-discipline $(r=.50)$, competence $(r=.45)$ and achievement $(r=.40)$. In addition to neuroticism facets, social support showed noteworthy correlations with trust $(r=.47)$, altruism $(r=.40)$, warmth $(r=$ $.46)$, and gregariousness $(r=.42)$, and positive emotion $(r=.48)$. Reflecting the modest incremental prediction provided by facets, there were only a few noteworthy semi-partial correlations. In particular, depression was an incremental predictor for most variables, which is consistent with it being a more pure measure of the negative affect that drives psychological symptoms and low social support. This partially supports the focus in Type D on negative affect as being a more refined measure than global 
neuroticism. However, there still remained many important facets beyond this, and in particular, there were strong correlations for a range of conscientiousness facets.

Table S1

Descriptive Statistics and Cronbach's Alpha Reliability for Personality Facets

\begin{tabular}{|c|c|c|c|}
\hline Facet & M & SD & $\alpha$ \\
\hline A1: Trust & 3.36 & 0.73 & .87 \\
\hline A2: Straightforwardness & 3.55 & 0.64 & .76 \\
\hline A3: Altruism & 4.00 & 0.49 & .74 \\
\hline A4: Compliance & 3.19 & 0.63 & .76 \\
\hline A5: Modesty & 3.58 & 0.64 & .80 \\
\hline A6: Tender-mindedness & 3.65 & 0.47 & .58 \\
\hline $\mathrm{C} 1:$ Competence & 3.55 & 0.49 & .62 \\
\hline $\mathrm{C} 2$ : Order & 3.14 & 0.59 & .67 \\
\hline C3: Dutifulness & 3.75 & 0.48 & .58 \\
\hline C4: Achievement & 3.31 & 0.61 & .75 \\
\hline C5: Self-discipline & 3.18 & 0.70 & .83 \\
\hline C6: Deliberation & 3.20 & 0.58 & .74 \\
\hline E1: Warmth & 3.75 & 0.65 & .83 \\
\hline E2: Gregariousness & 3.02 & 0.73 & .80 \\
\hline E3: Assertiveness & 2.97 & 0.65 & .79 \\
\hline E4: Activity & 3.04 & 0.57 & .65 \\
\hline E5: Excitement-seeking & 3.18 & 0.61 & .60 \\
\hline E6: Positive Emotion & 3.54 & 0.72 & .82 \\
\hline N1: Anxiety & 3.19 & 0.73 & .82 \\
\hline N2: Angry Hostility & 2.79 & 0.70 & .81 \\
\hline N3: Depression & 3.03 & 0.85 & .86 \\
\hline N4: Self-consciousness & 3.16 & 0.67 & .73 \\
\hline N5: Impulsiveness & 3.32 & 0.58 & .69 \\
\hline N6: Vulnerability & 2.63 & 0.61 & .78 \\
\hline O1: Fantasy & 3.47 & 0.62 & .74 \\
\hline O2: Aesthetics & 3.25 & 0.75 & .81 \\
\hline O3: Feelings & 3.78 & 0.54 & .71 \\
\hline O4: Actions & 3.01 & 0.50 & .58 \\
\hline O5: Ideas & 3.60 & 0.74 & .85 \\
\hline O6: Values & 3.90 & 0.48 & .65 \\
\hline
\end{tabular}


Table S2

Zero-Order Correlations and Semi-Partial Correlations (Controlling for the Big Five Factors) between Personality Facets and Health Outcomes

\begin{tabular}{|c|c|c|c|c|c|c|c|c|c|c|}
\hline \multirow[b]{3}{*}{ A1: Trust } & \multicolumn{5}{|c|}{ Zero-order $\mathrm{r}$} & \multicolumn{5}{|c|}{ Semi-partial $\mathbf{r}$} \\
\hline & $\mathrm{HB}$ & SS & PHYS I & PSYCH & $\begin{array}{l}\text { Mean } \\
\text { Abs } r\end{array}$ & $\mathrm{HBS}$ & SS & PHYS $\quad \mathrm{F}$ & PSYCH & $\begin{array}{l}\text { Mean } \\
\text { Abs } r\end{array}$ \\
\hline & .22 & .47 & -.19 & -.37 & $\mathbf{3 1}$ & .11 & .11 & -.01 & -.04 & .07 \\
\hline A2: Straightforwardness & .10 & .23 & -.02 & -.19 & .14 & .03 & -.04 & .04 & -.04 & .04 \\
\hline A3: Altruism & .19 & .40 & -.07 & -.16 & .20 & .06 & .07 & .01 & .01 & .04 \\
\hline A4: Compliance & -.01 & .23 & .01 & -.10 & .09 & -.07 & .00 & .02 & .07 & .04 \\
\hline A5: Modesty & -.10 & .02 & .17 & .08 & .09 & -.03 & -.06 & .07 & .05 & .05 \\
\hline A6: Tender-mindedness & -.12 & .06 & -.03 & .00 & .05 & -.12 & -.09 & -.15 & -.05 & .10 \\
\hline C1: Competence & .45 & .34 & -.25 & -.30 & .33 & .05 & .08 & .02 & -.05 & .05 \\
\hline C2: Order & .35 & .26 & -.14 & -.12 & .22 & -.05 & .02 & .08 & .09 & .06 \\
\hline C3: Dutifulness & .37 & .28 & -.23 & -.27 & .29 & -.02 & -.01 & -.06 & -.07 & .04 \\
\hline C4: Achievement & .41 & .19 & -.22 & -.15 & .24 & .03 & -.06 & -.03 & -.01 & .03 \\
\hline C5: Self-discipline & .50 & .38 &.- .38 & -.43 & .42 & .04 & -.05 & -.09 & -.09 & .07 \\
\hline C6: Deliberation & .27 & .16 & -.10 & -.09 & .15 & -.04 & .04 & .05 & .10 & .06 \\
\hline E1: Warmth & .19 & .46 & -.17 & -.26 & .27 & .00 & .03 & .03 & .00 & .02 \\
\hline E2: Gregariousness & .19 & .42 & -.21 & -.23 & .26 & -.01 & .02 & .02 & .09 & .04 \\
\hline E3: Assertiveness & .24 & .22 & -.21 & -.18 & .21 & -.03 & -.06 & .07 & .08 & .06 \\
\hline E4: Activity & .34 & .30 & -.23 & -.25 & .28 & .05 & -.06 & .03 & .00 & .04 \\
\hline E5: Excitement-seeking & -.03 & .09 & -.09 & .00 & .05 & -.03 & -.01 & -.08 & -.01 & .03 \\
\hline E6: Positive Emotion & .25 & .48 & -.29 & -.41 & .36 & .02 & .09 & -.05 & -.17 & .09 \\
\hline N1: Anxiety & -.22 & -.32 & .41 & .68 & .41 & .03 & .11 & .03 & .17 & .08 \\
\hline N2: Angry Hostility & -.20 & -.39 & .25 & .46 & .33 & .02 & .03 & -.08 & -.09 & .05 \\
\hline N3: Depression & -.41 & -.53 & .51 & .72 & .54 & -.11 & -.17 & .12 & .21 & .15 \\
\hline N4: Self-consciousness & -.23 & -.38 & .37 & .47 & .36 & .06 & .01 & -.06 & -.12 & .06 \\
\hline N5: Impulsiveness & -.37 & -.26 & .33 & .33 & 32 & -.13 & .04 & .01 & -.19 & .09 \\
\hline N6: Vulnerability & -.24 & -.38 & .38 & .56 & .39 & .14 & -.02 & -.03 & .03 & .05 \\
\hline O1: Fantasy & -.08 & -.16 & .04 & .13 & .10 & -.03 & -.06 & .00 & .03 & .03 \\
\hline O2: Acsthetics & .13 & .04 & .00 & .01 & .05 & .02 & -.04 & .07 & .01 & .03 \\
\hline O3: Feelings & .13 & .11 & -.02 & .07 & .08 & -.01 & .00 & -.03 & -.02 & .02 \\
\hline O4: Actions & .19 & .18 & -.16 & -.22 & .19 & .14 & .05 & -.06 & -.09 & .09 \\
\hline O5: Ideas & .10 & -.01 & .03 & .10 & .06 & -.01 & .01 & .09 & .09 & .05 \\
\hline O6: Values & -.03 & .12 & -.07 & -.01 & .06 & -.10 & .05 & -.10 & -.05 & .08 \\
\hline Mean Abs r & .22 & .26 & .19 & .25 & .23 & .05 & .05 & .05 & .07 & .06 \\
\hline
\end{tabular}

Overall, narrow traits provided a small improvement in prediction over and above the Big Five. In general, previous research has commonly obtained increases in prediction of 10 to 50 percent (Anglim \& Grant, 2014, 2016), and the obtained increase in this study was at the lower end of this range. Nonetheless, the relative size of correlations with narrow traits did help to explain relationships between personality and health-related variables. For example, self-discipline was the conscientiousness facet with the largest correlation with health behaviors, and warmth, gregariousness, and positive emotions were the largest correlating 
facets of extraversion with social support. In some respects, the subscales of Type D have a similar breadth to some of the narrow facets of the NEO-PI-R. Interestingly, the narrow facet of depression was superior to negative affectivity in its average prediction, and outside of the neuroticism domain, the conscientiousness facet of self-discipline had slightly better prediction than social inhibition. Thus, overall, negative affectivity and social inhibition are two narrow traits that provide reasonable prediction of health-related variables, but a comprehensive personality framework provides more perspective on the relative importance of different narrow aspects of personality.

\subsection{Cut-off Analysis}

The default cut-scores for Type D personality may not be optimal from a predictive validity perspective. Thus, to examine whether alternative cut-offs resulted in improved prediction, we generated categorical Type D variables using all possible cut-offs. We then correlated each categorical Type D variable with the four outcome measures and used the mean absolute correlation with these four outcomes as a measure of predictive validity. Figure S1 presents a contour plot showing the achieved mean absolute correlation for different cut-offs. It shows that optimal cut-offs were achieved when NA cut-offs were between 12 and 18, and SI cut-offs were less than 11 .

This general finding is consistent with NA being the stronger predictor of all but social support. As such, similar prediction could be achieved by relying entirely on an cut-off on NA, as occurs when the cut-off for SI is zero, and is approximated when SI is low.

While a broad range of cut-offs yielded similar predictive validity, the optimal value in the current sample involved cut-offs of 15 for NA and 9 for SI. This yielded a mean absolute R of .45. This compares to .37 using the standard cut-offs of 10 and 10, and .50 when using continuous Type D.

Nonetheless, we assume that these specific cut-offs are partially sample specific. While the present sample size is reasonable for most purposes, using a sample-optimized set of cutoff scores will provide a biased estimate of the predictive validity of the optimum cut-off. More generally, the optimal cut-off may be contingent on the distributional features of the sample. Thus, we are not advocating the use of alternative cut-offs. Instead, this analysis merely provides a general robustness check on the observation that relatively-continuous measures of personality traits provide superior prediction. 


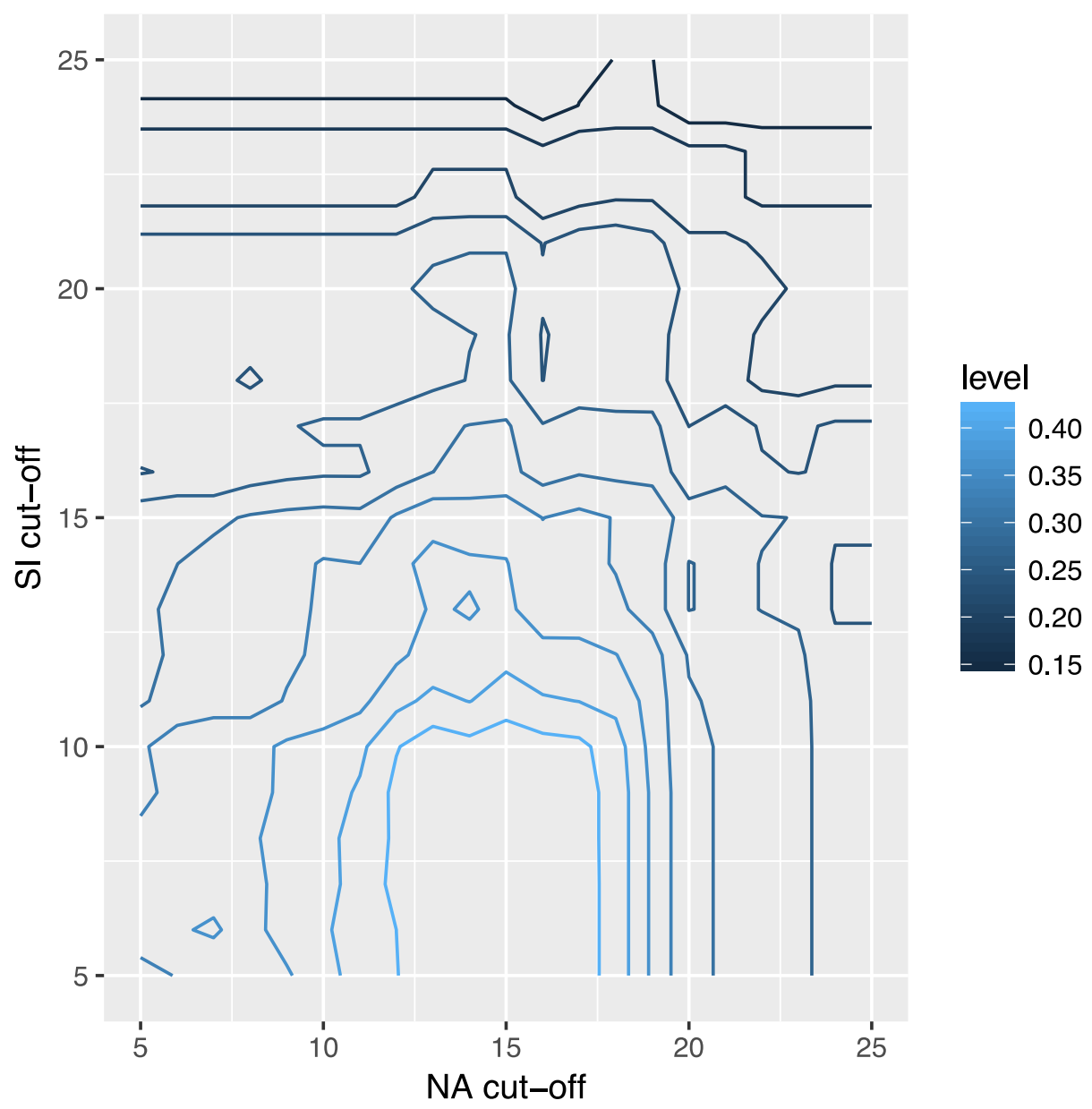

Figure S1. Contour plot showing mean absolute correlation between Type D categorical and the four outcome measures using varying NA and SI cut-offs for generating the Type D categorical variable. 